\title{
EVALUATION OF OPTICALLY ILLUMINATED MOSFET CHARACTERISTICS BY TCAD SIMULATION
}

\author{
Prerana Jain ${ }^{1}$ and Mishra B.K ${ }^{2}$ \\ ${ }^{1}$ SKVM's NMIMS,Vile Parle(W),Mumbai,India \\ jainpnjegmail.com \\ ${ }^{2}$ Principal, Thakur College of Engg and Technology, Mumbai \\ drbk.mishra@thakureducation.org
}

\begin{abstract}
In this paper we report effect of optical illumination on Silicon MOSFET. The MOSFET has been studied in respect of current voltage, transconductance admittance and scattering parameters. Gain analysis of the Silicon MOSFET is done in dark and under optical illumination. The device is fabricated using ATHENA process simulator and the device simulation is performed using ATLAS ${ }^{\mathrm{TM}}$ from SILVACO international. The simulation results indicate potential of MOSFET as optically sensitive structure which can be used for increase in data transmission/reception rates, reduction of interconnect delays, elimination of clock skew, or as a photodetector for optoelectronic applications at low and radio frequency.
\end{abstract}

\section{KEYWORDS}

AC, DC, MOSFET, Optical Illumination, RF, Simulation.

\section{INTRODUCTION}

Silicon CMOS technology has matured into the nanometre regime. The feasibility of RF CMOS circuit integration has clearly been demonstrated due to reduction in gate length [1]. This continued transistor miniaturization is the key for sustained performance improvement. But this aggressive scaling is also associated number of higher order effects which significantly affect the device operations. The design of RF CMOS circuits in practical systems is a real challenge due to the strong constraints on power consumption and noise. This leaves very little margin for design[2]. This necessitates advances in materials, device structures and other alternatives to gain better control over the device characteristics. In spite of superior performance of compound semiconductors, the cost issue in silicon and conventional CMOS technology devices dominates the market place[3][4]. One of the alternatives is use of optical port to enhance the device characteristic. Optoelectronic and CMOS integration offers all the advantages like immunity to electromagnetic interference, better reliability etc [5]. Properties of Silicon offer possibility of integration on the same chip. Electronic circuits, photonic circuits and micromechanical structures can be fabricated at low cost and high reproducibility using Silicon technologies[6][7]. Silicon detectors are also appropriate for the visible and near infrared spectral range[ 8$]$. Several photodetector structures like photoconductors, PIN diodes and avalanche photo diodes(APD) are available. Photoconductors and PIN diodes have no internal gain and require high are power. The APD's offer high gain but suffer from high amplification noise problem. Transistor photodetectors offer advantage of gain in comparison with diode detector structures and hence a MOSFET under illuminated condition is considered for analysis [9].

In a device, the I-V characteristics are important at DC while Scattering (S) parameters and admittance (Y) parameters are important at high frequency. $\mathrm{Y}$ and $\mathrm{S}$ parameters are small signal parameters by definition and high frequency behaviour of the device is determined around a bias 
point over its operational bandwidth[10]. The $\mathrm{S}$ parameters are also important as they are used to determine signal power gain and various figures of merit[11] .

Technology computer-aided design (TCAD) software is powerful tool and is widely used to study, optimize and predict the behaviour of devices. Characterization of devices under effects of various electrical, thermal and optical conditions is also possible [12].This paper presents analysis of MOSFET for DC and AC under small signal condition for frequency range of $1 \mathrm{GHz}$ to 10 GHz. The gain and feedback considerations are of prime importance while dealing with active devices and hence power gain issues and their graphical displays are the starting point of analysis and design of high frequency amplifiers. To meet this requirement the transducer gain, available power gain, operating power gain, unilateral power gain and maximum stable power gain are evaluated for dark and under optical radiation. Current gain analysis of the device is also presented. Simulations are done for MOSFET under dark and optically illuminated condition. All the calculations presented in this work have been obtained using ATLAS from SILVACO ${ }^{\circledR}$ international unless stated otherwise. The results indicate the prospective of the device as promising candidate for optoelectronic applications at low as well at RF frequency.

The paper is structured as follows. Section -II presents MOSFET formation in TCAD, the device parameters, models and the methodology used for simulation under dark condition. Section III considers MOSFET under illuminated conditions. Section IV is devoted for results and discussion arising out of simulations.

\section{THEORY}

The schematic of the structure under consideration is a lightly doped drain (LDD) N channelMOSFET as shown in figure-1.

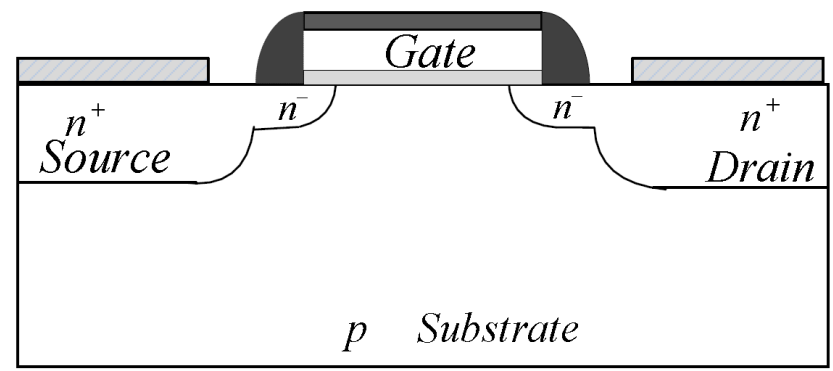

Figure 1. Schematic of LDD MOSFET

The conventional structure of MOSFET needs to be modified at RF to achieve the requirement of figures of merit like low noise figure, transit frequency $\mathrm{f}_{\mathrm{T}}$ and maximum frequency of oscillation $\mathrm{f}_{\max }$. Several modifications in the typical bulk structure have been proposed for MOSFET at RF [4]. One of the structure is a multifinger MOSFET, which is modelled and analysed by Jain et al.[13][14][15]. Another proposed device structures considered for present analysis is standard LDD MOSFET.

This structure is fabricated using ATHENA which provides capability of numerical, physicallybased, two-dimensional simulation of semiconductor processing. The fabrication process in ATHENA started with selection of wafer and completed with metallization for electrodes for a standard LDD MOS process [16].The parameters for the device were estimated by use of EXTRACT command. The outcome of the fabrication process simulation is the device structure as indicated in Figure 2 and Figure 3. Figure 2 is a NMOSFET structure with materials and junctions simulated in ATHENA. Figure 3 is the structure with net doping profile of the NMOSFET. 
International Journal of VLSI design \& Communication Systems (VLSICS) Vol.4, No.2, April 2013

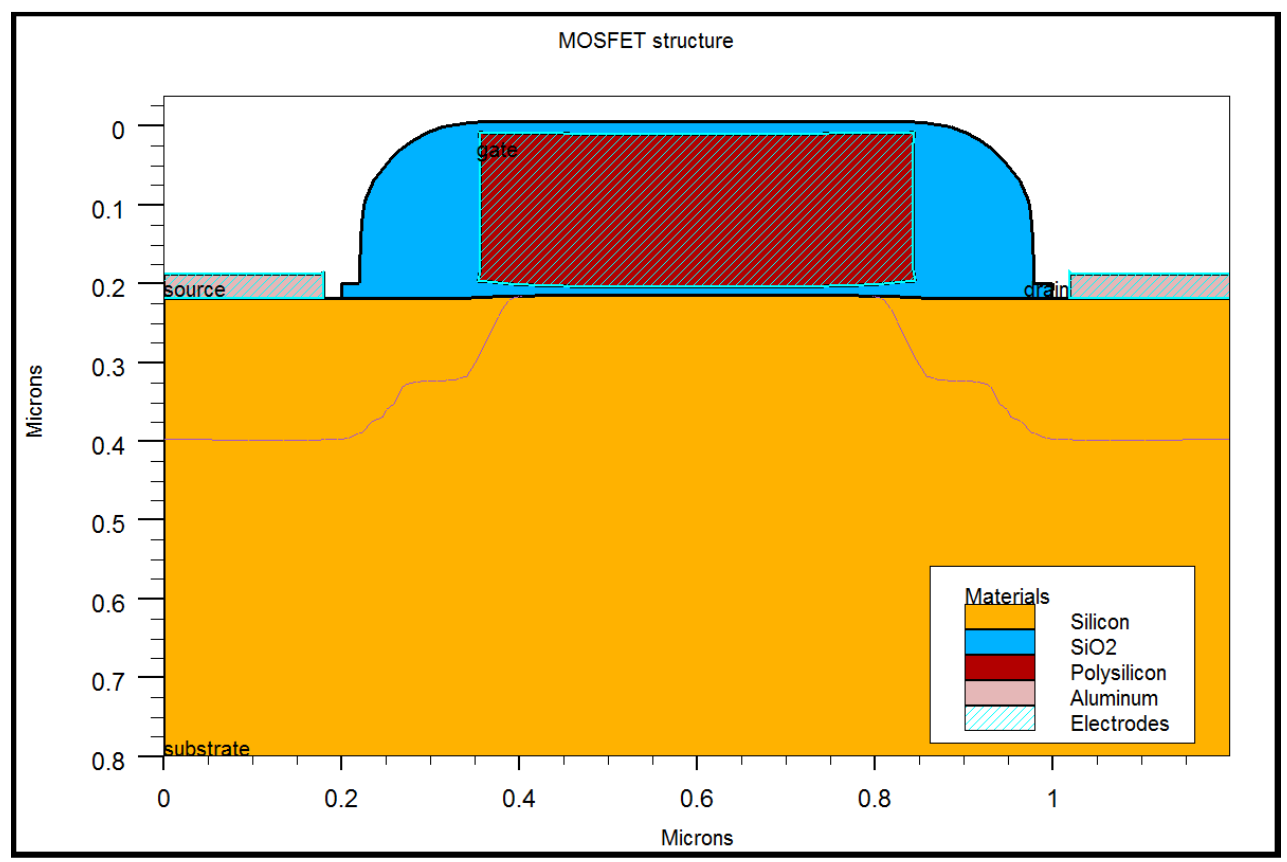

Figure 2. MOSFET structure with materials and junctions in TCAD

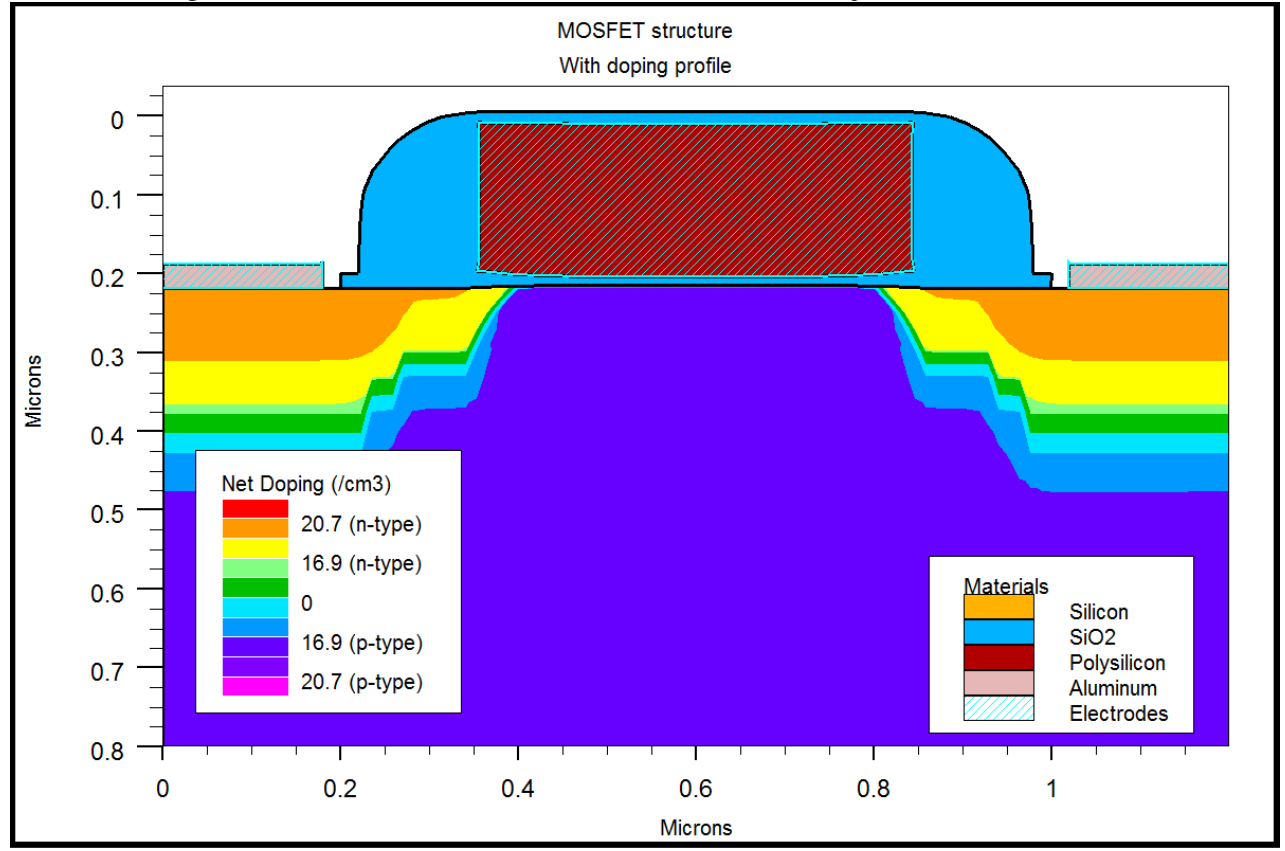

Figure 3.MOSFET structure with net doping profile.

The NMOSFET simulated uses a $\mathrm{p}$ type substrate of concentration of $1 \times 10^{17}$ atoms $/ \mathrm{cm}^{3}$ with an ion implanted profile. The surface concentration under the channel is $3.74188 \times 10^{16}$ atoms $/ \mathrm{cm}^{3}$. The gate oxide is $10 \mathrm{~nm}$ thick deposited by dry oxidation process. The fabricated junction depth achieved is about $0.17 \mu \mathrm{m}$. The source and drain structures have been processed to achieve LDD structure. The drain and source regions have peak concentration of $5.36 \times 10^{20}$ atoms $/ \mathrm{cm}^{3}$ and the lightly doped region has concentration of $2 \times 10^{17}$ atoms $/ \mathrm{cm}^{3}$. The gate is made of $\mathrm{n}$ doped polysilicon while the source and drain electrodes are of Aluminium. The gate length of the device is $0.36 \mu \mathrm{m}$. The long channel threshold voltage of the fabricated structure extracted is $0.6 \mathrm{~V}$. The 
device fabrication is done using two dimensional simulator, and hence the device width is $1 \mu \mathrm{m}$ unless specified.

Atlas is two and three dimensional physically based device simulator. Simulation in ATLAS requires appropriate mesh definition. It has been demonstrated that inappropriate mesh spacing often affects accuracy of simulation. The mesh structure of MOSFET is presented in figure 3. Lateral spacing in the channel under the gate and source drain region is small. Similarly very small vertical mesh spacing is selected in the channel under the gate for optimized simulation considering speed and accuracy of simulation.

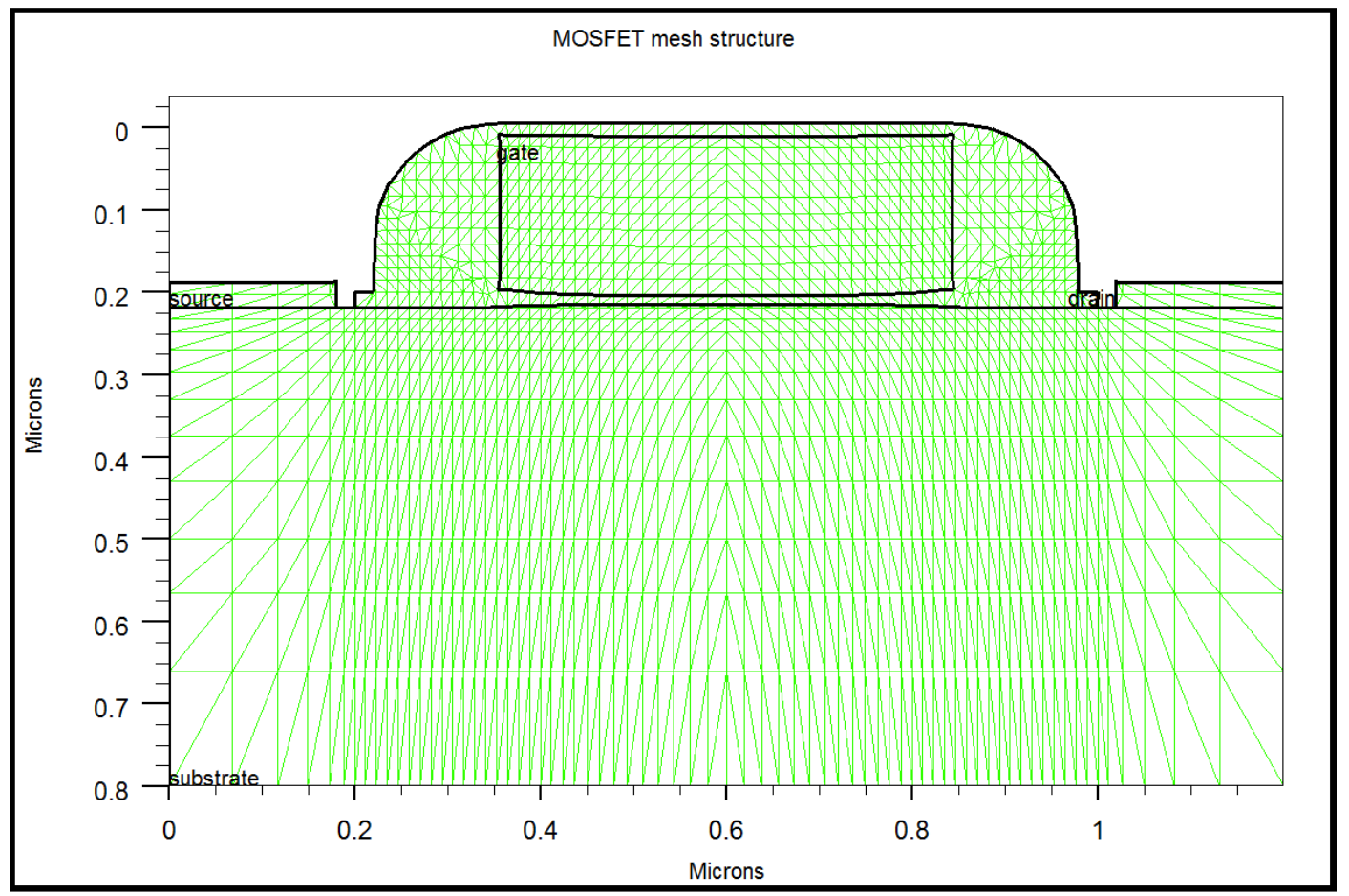

Figure 4. Mesh structure of MOSFET

The numerical simulation of MOSFET is carried out using MOS models in the ATLAS. MOS parameter enables Shockley-Read-Hall (SRH), Fermi Statistics (FERMI), and the Lombardi Mobility model (CVT) for transverse field dependence. The CVT model sets a general purpose mobility model to include concentration, temperature, parallel field and transverse field effects. The SRH model accounts for recombination effects, and uses fixed minority carrier lifetimes. The contact statement is used to define gate electrode which is $n$ doped poly silicon for which the simulator assumes work function of $4.7 \mathrm{eV}$.For accurate simulation of MOS devices the interface charge at the oxide, must be considered. This is done by setting the QF parameters for the INTERFACE statement to a value of $3 \times 10^{10} / \mathrm{cm}^{2}$ [17]

Several numerical methods are available and can be used for calculating the solutions to semiconductor device[18]. In the ATLAS tool a method or combination of methods is selected depending on the equations to be solved. For the MOS structure the trend is to simulate only majority carriers to improve speed of simulation. But this is not advised when the simulation involves small signal ac analysis or when recombination effects are to be considered. To calculate electrical parameters, the simulator makes use drift diffusion formulation discretized over a multidimensional numerical mesh[19]. The isothermal drift diffusion model requires the solution of three decoupled equations for potential, electron concentration, and hole concentration. 
This solution was achieved by use of Gummel Newton iteration scheme. This method initially performs a GUMMEL iteration to obtain an improved initial guess for the NEWTON solution scheme. This is slow but reliable way of obtaining solutions for any device[20]. For device analysis under any applied bias, first initial solution must be performed with zero bias for initial guess to determine potential and carrier concentrations from the doping profile. The simulator solves the three basic equations using drift diffusion model evaluating for potential, electron concentration and hole concentration with appropriate assumptions and calculates the drain current[21].

The drain current under dark condition was evaluated by ramping the bias in small initial steps. The ATLAS tool calculates the drain current under varying gate bias and drain bias, but the transconductances cannot be obtained directly. TONYPLOT ${ }^{\mathrm{TM}}$ is used which is a graphical post processing tool for use with all SILVACO simulators. The transconductances are obtained graphically using TONYPLOT functions from the plot of drain current with respect to gate and drain voltages by taking derivatives as per (1). The gate transconductance $g_{m}$ and the drain conductance $g_{d s}$ are given by

$$
\begin{aligned}
& g_{m g}=g_{m}=\left[\frac{\partial I_{D S}}{\partial V_{G B}}\right] V_{S}, V_{D}=\text { const } \\
& g_{m d}=g_{d s}=\left[\frac{\partial I_{D S}}{\partial V_{D B}}\right] V_{G}, V_{D}=\text { const }
\end{aligned}
$$

The ac analysis is performed to determine small signal characteristics of the device. Instead of extracting a small-signal model from measurements, the complete small-signal model of the intrinsic device is derived from physically-based simulations. Several advantages can be achieved by use of this methodology. Errors resulting from measurements and extraction of extrinsic component are decoupled; Non Quasi Static (NQS) effects are included by default resulting in a good description of the electrical performance, even at frequencies beyond transition frequency $\mathrm{f}_{\mathrm{T}}$. Parameter extraction between any two ports of the device is easily feasible which is difficult otherwise [22].

The ATLAS tool supports calculation of various AC parameters like Hybrid (H), Admittance(Y), Impedance (Z), Scattering(S) etc. The gain analysis can also be done as a part of small signal analysis. AC sinusoidal small-signal analysis should be performed after solving for a DC condition. The full Newton method must be used for this analysis. Frequency range of interest, device width and the method for ac analysis has to be selected. A direct parameter is included which is robust for all range of frequencies. The gate terminal is selected as input port where ac bias will be applied, while drain terminal is selected as output port indicating that device works in common source configuration.

Design of high frequency circuits is often done with help of $\mathrm{Y}$ parameters. The device simulator does not make a distinction between the intrinsic and parasitic parts of the device. The $\mathrm{Y}$ parameters are simulated using the (2) for each DC bias condition as in[11].

$$
-y_{j k}=g_{j k}+j \omega c_{j k}
$$

The $y_{j k}$ parameters are obtained by applying a small-signal voltage at the $k^{\text {th }}$ terminal and detecting current at the $j^{\text {th }}$ terminal while all other terminals are AC grounded with following relationship 


$$
y_{j k}=\frac{i_{j}}{v_{k}}
$$

Admittance or impedance parameters require short circuit or open circuit condition for measurement, which is difficult to achieve at high frequency where lead inductance and parasitic capacitances dominate the measurement. [13].Scattering or $\mathrm{S}$ parameters are alternative to admittance or impedance parameters for characterization at high frequency. S parameters are relatively simple to measure, conceptually simple, analytically convenient and capable of providing a great insight into measurement or design problem. The $\mathrm{S}$ parameters can be obtained from $Y$ parameters using $Y$ to $S$ conversion for the desired characteristics impedance as in[23,24]. The expressions for gain and unilateral figure of merit are obtained from $\mathrm{S}$ parameters and reflection coefficients. The source $\left(\Gamma_{S}\right), \operatorname{load}\left(\Gamma_{L}\right), \operatorname{input}\left(\Gamma_{\text {in }}\right)$ and output $\left(\Gamma_{\text {out }}\right)$ reflection coefficients have same definitions as in [23].

The transducer power gain $G_{T}$, quantifies the gain of the amplifier placed between source and load.

$$
G_{T}=\frac{\left(1-\left|\Gamma_{L}\right|^{2}\right)\left|S_{21}\right|^{2}\left(1-\left|\Gamma_{S}\right|^{2}\right)}{\left|\left(1-S_{11} \Gamma_{S}\right)\left(1-S_{22} \Gamma_{L}\right)-S_{21} S_{12} \Gamma_{L} \Gamma_{S}\right|^{2}}
$$

An often employed approximation for the transducer power gain is the so-called unilateral power gain, $G_{T U}$, which neglects the feedback effect of the amplifier $\left(\mathrm{S}_{12}=0\right)$. It is often used as a basis to develop approximate designs for an amplifier and its input and output matching networks.

The available power gain $G_{A}$ for load side matching $\left(\mathrm{T}_{\mathrm{L}},=\right.$ Tout $)$ is defined as

$$
G_{A}=\frac{\left|S_{21}\right|^{2}\left(1-\left|\Gamma_{S}\right|^{2}\right)}{\left(1-\left|\Gamma_{\text {out }}\right|^{2}\left|1-S_{11} \Gamma_{S}\right|^{2}\right.}
$$

The operating power gain is the ratio of the power delivered to the load to the power supplied to the amplifier.

The current gain $h_{21}$ of a small-signal common-source amplifier stage is defined as

$$
h_{21}=\frac{\mathrm{I}_{1}}{\mathrm{I}_{1}} \mid V_{2}=0=\frac{\mathrm{Y}_{21}}{\mathrm{Y}_{11}}
$$

The calculation of current gain $h_{21}$ is important as it can be used to evaluate frequency $\mathrm{f}_{\mathrm{t}}$ Transition frequency $f_{t}$ also known as unity gain frequency and is one of the figure of merit for RF operation and indicates the frequency at which current gain $h_{21}$ equals one.

$$
G_{T U}=\frac{\left(1-\left|\Gamma_{L}\right|^{2}\right)\left|S_{21}\right|^{2}\left(1-\left|\Gamma_{S}\right|^{2}\right)}{\left|1-\Gamma_{L} S_{22}\right|^{2}\left|1-S_{11} \Gamma_{S}\right|^{2}}
$$

\section{MOSFET UNDER ILLUMINATION}

When light falls on the surface of a semiconductor material, photons whose energy is in greater than that of the band-gap energy of the semiconductor material, are absorbed. This absorption excites an electron to jump into the conduction band and as a result a hole is left in the valence band. These promoted electrons and valence band holes behave as free particles and travel under intrinsic or externally-applied electric field. This continuous separation of electron-hole pairs due 
to the absorption of photons enhances the conductivity. This photocurrent is directly proportional to the intensity of the incident light.

The schematic of MOSFET under illumination is as shown in figure 5. The Aluminium electrodes block the incident light, but the light can be absorbed at the edge of the gate electrodes. This modifies the depletion region width of the drain $\left(\mathrm{Y}_{\mathrm{dD}}\right)$ and source $\left(\mathrm{Y}_{\mathrm{dS}}\right)$ region and hence the depletion region width at gate $\left(\mathrm{Y}_{\mathrm{dG}}\right)$. Silicon is sensitive to $\mathrm{UV}$, visible and near-infrared regions i.e. from around $200 \mathrm{~nm}$ to $1100 \mathrm{~nm}$ of wavelength. The peak response can be modified to suit specific applications using special antireflection coatings and filters [9] In the present work no such modification has been done.

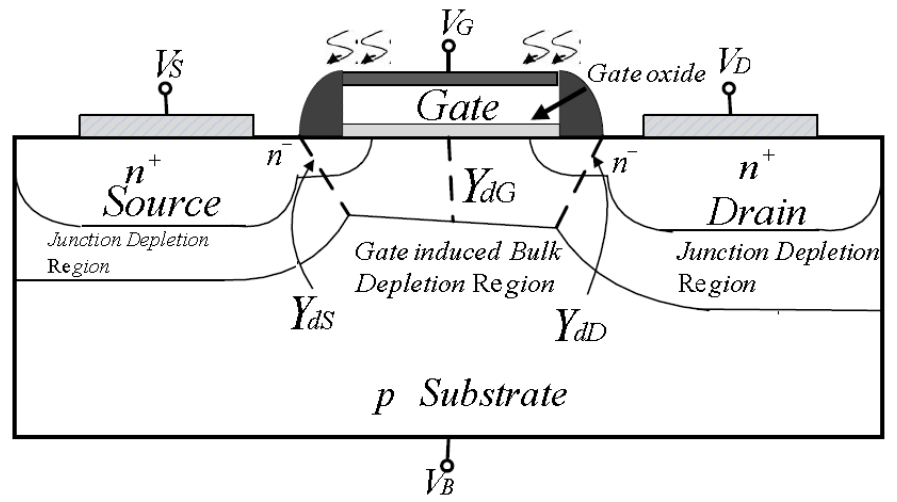

Figure 5. Schematic of MOSFET under illumination.

The ATLAS framework includes LUMINOUS which is optoelectronic simulator. LUMINOUS is a general purpose ray trace and light absorption program which calculates optical intensity profiles within the semiconductor device, and converts these profiles into photo generation rates. This unique coupling of tools allows simulation of electronic responses to optical signals for a broad range of optical detectors. For the simulation purpose an optical source originates $0.5 \mathrm{um}$ above the device, perpendicular to the front surface of the device is considered. The wavelength of the source is $830 \mathrm{~nm}$ with intensity of $0.25 \mathrm{~mW} / \mathrm{cm}^{2}$ assumed to be incident over the MOSFET. Presently, it has been found extensively that lot of systems for home-networking and chip-tochip interconnect make use of wavelength less than $850 \mathrm{~nm}$ and hence is chosen for simulation.[8]

The SRH, CONMOB, AUGER and FLDMOB models activated for simulation. The effect of the transmission coefficients, reflection coefficients, along with the integrated loss due to absorption over the ray path is considered for each ray. This computation due to the cumulative effect is saved for each ray. The generation associated with each grid point can be calculated by integration of the generation rate formula as in equation (5). The calculation for the generation rate is then performed over the area of intersection between the ray and the polygon associated with the grid point.

$$
G=\eta_{0} \frac{P^{*} \lambda}{h c} \alpha e^{-\alpha y}
$$

Where

$P^{*}$ contains the combined effects of loss due to absorption, reflections and transmissions.

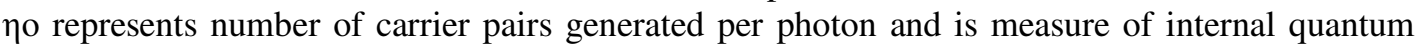
efficiency

$y$ is a relative distance for the ray.

$h$ is Planck's constant 
$\lambda$ is the wavelength.

$c$ is the speed of light.

$\alpha$ is the absorption coefficient.

The simulator calculates source photo current $I_{S}$ and available photocurrent $I_{A}$. The source photo current can be considered as a measure of the rate of photons incident on the device expressed as a current density and is given by(6).

$$
I_{s}=q \frac{B_{n} \lambda}{h c} W_{t}
$$

The available photocurrent can be thought of as a measure of the rate of photo absorption in the device expressed as a current density. This is less than the source photocurrent. The losses are due to reflection and transmission of light out of the device structure.

$$
I_{A}=q \frac{B_{n} \lambda}{h c} \sum_{i=1}^{N_{R}} W_{R} \int_{01}^{x_{i}} P_{i} \alpha_{i} e^{-\alpha_{i} y} d y
$$

Where

$B_{n}$ is the intensity in beam number $n$, $\lambda$ is the source wavelength,

$h$ is Planck's constant, $c$ is the speed of light,

$\mathrm{W}_{\mathrm{t}}$ is the width of the beam including the effects of clipping,

$\mathrm{N}_{\mathrm{R}}$ is the sum is taken over the number of rays traced,

$W_{R}$ is the width associated with the ray,

$Y i$, is the integral is taken over the length, associated with the ray,

$P i$ accounts for the attenuation

$\alpha^{i}$ is the absorption coefficient in the material that the ray is traversing,

To account for the diffraction or coherent effects beam propagation method is used.The total current under illumination is the addition of dark current and the current due the carriers generated due to photons.

\section{RESULTS AND DISCUSSION}

The device under consideration is a Silicon N-MOSFET with a LDD structure. The gate length is $0.36 \mu \mathrm{m}$ and the width is $1 \mu \mathrm{m}$ for DC analysis. The drain current is calculated by varying gate and drain voltage in small steps, one at a time, since the equations are solved by numerical methods. The simulation has been performed for the device in dark condition and also under optical illumination for incident wavelength of $830 \mathrm{~nm}$ and beam intensity of $0.25 \mathrm{~mW} / \mathrm{cm} 2$.

Figure $6 \mathrm{~A}$ indicates plot of drain current with respect to gate voltage $\mathrm{V}_{\mathrm{G}}=1 \mathrm{~V}, 2 \mathrm{~V}$ and $3 \mathrm{~V}$ in dark condition and $\mathrm{V}_{\mathrm{D}}$ varying from $0 \mathrm{~V}$ to $3 \mathrm{~V}$. Figure $6 \mathrm{~B}$ is plot for similar bias conditions of gate and drain voltage, under optical illumination. Figur e 7A and 7B are comparative plots of drain current with respect to gate voltage $\mathrm{V}_{\mathrm{G}=} 1 \mathrm{~V}$ and $\mathrm{V}_{\mathrm{G}=} 2 \mathrm{~V}$ in dark and illuminated condition. 


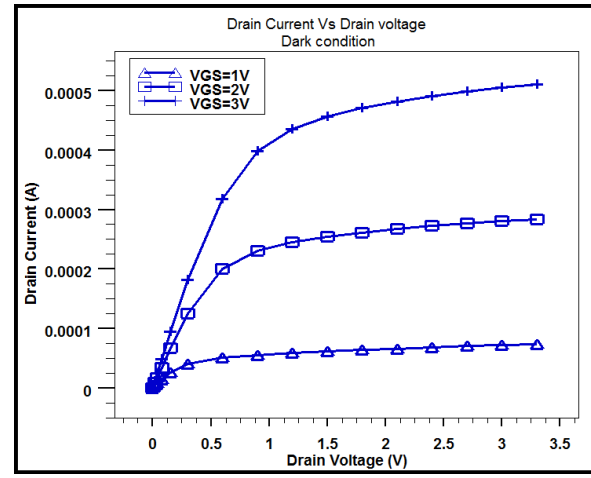

Figure 6

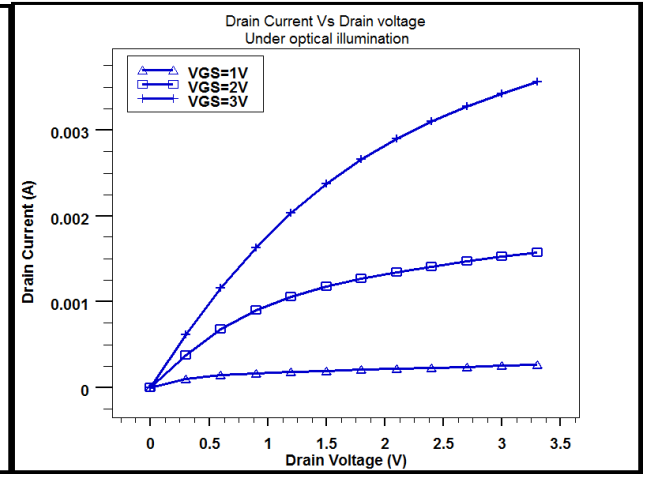

Figure 6.2

Figure 6A:Drain current $\mathrm{Vs}$ Drain voltage for $\mathrm{V}_{\mathrm{G}}=1 \mathrm{~V}, 2 \mathrm{~V}$ and $3 \mathrm{~V}$ in dark condition Figure 6B: Drain current Vs Drain voltage for $\mathrm{V}_{\mathrm{G}}=1 \mathrm{~V}, 2 \mathrm{~V}$ and $3 \mathrm{~V}$ under optical illumination.

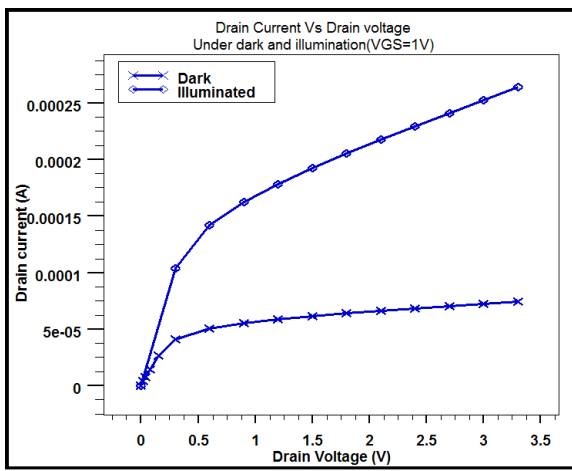

Figure 7(A)

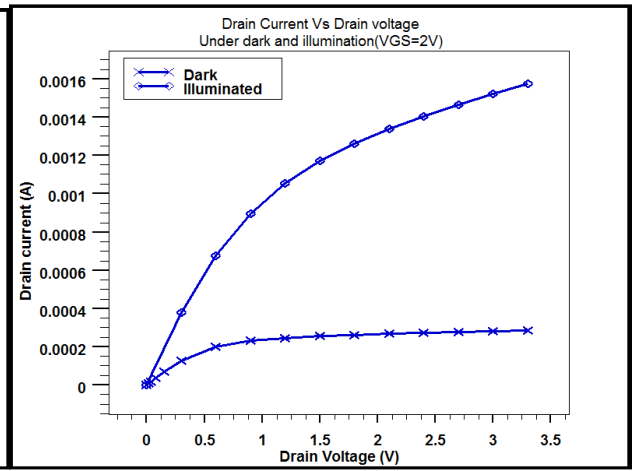

Figure 7(B)

Figure 7A: Drain current Vs Drain voltage for $\mathrm{V}_{\mathrm{G}}=1 \mathrm{~V}$ in dark \& under optical illumination. Figure 7B: Drain current Vs Drain voltage for $\mathrm{V}_{\mathrm{G}}=2 \mathrm{~V}$ in dark \& under optical illumination.

It can be seen that there is appreciable increase in drain current under optical illumination for varying gate voltage $\mathrm{V}_{\mathrm{G}}$ as compared to dark condition. This is because incident radiation results in excess electron hole pair generation in the depletion region. Because of this, photo voltage $\left(\mathrm{V}_{\mathrm{OP}}\right)$ is developed modifying the effective gate bias to $\mathrm{V}_{\mathrm{G}}+\mathrm{V}_{\mathrm{OP}}$. This higher effective gate bias enhances the device conductivity and hence the drain current increases.

Figure $8 \mathrm{~A}$ and Figure $8 \mathrm{~B}$ are plots of drain current with respect to varying gate voltage $\mathrm{V}_{\mathrm{G}}$ from 0 $\mathrm{V}$ to $3 \mathrm{~V}$ with $\mathrm{V}_{\mathrm{D}}=0.1 \mathrm{~V}$ and $1 \mathrm{~V}$ in dark and under optical radiation. Again here similar effect is observed that drain current increases under light. Conventionally drain current-gate voltage characteristics are used to evaluate threshold voltage. It can be seen that the effective threshold voltage reduces under influence of optical radiation confirming that photovoltage develops across the gate. The effect of optical radiation is more pronounced with increasing drain bias from $0.1 \mathrm{~V}$ to $1 \mathrm{~V}$ for constant gate voltage because the depletion width of the MOSFET is affected by drain bias. 


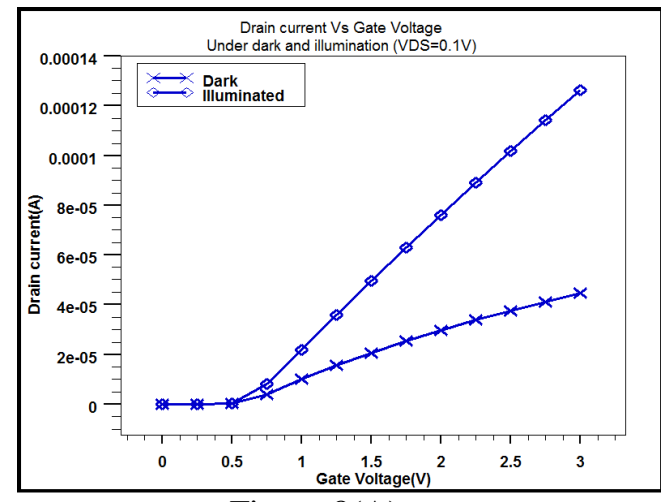

Figure 8(A)

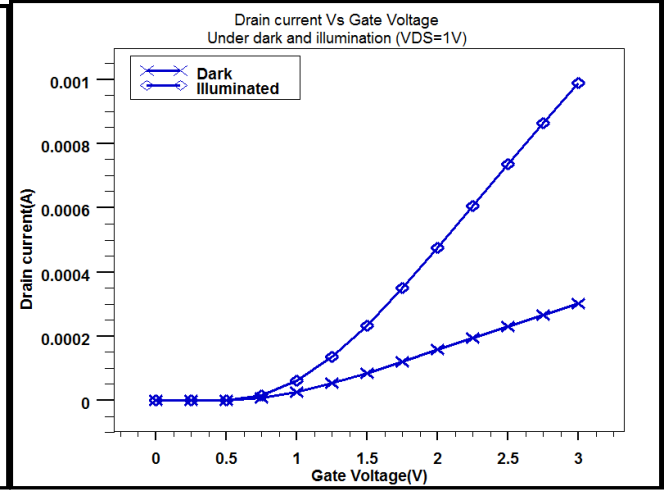

Figure 8(B)

Figure 8A: Drain current Vs Gate voltage for $\mathrm{V}_{\mathrm{D}}=0.1 \mathrm{~V}$ in dark \& under optical illumination. Figure 8B: Drain current $V s$ Gate voltage for $V_{D}=1 \mathrm{~V}$ in dark \& under optical illumination

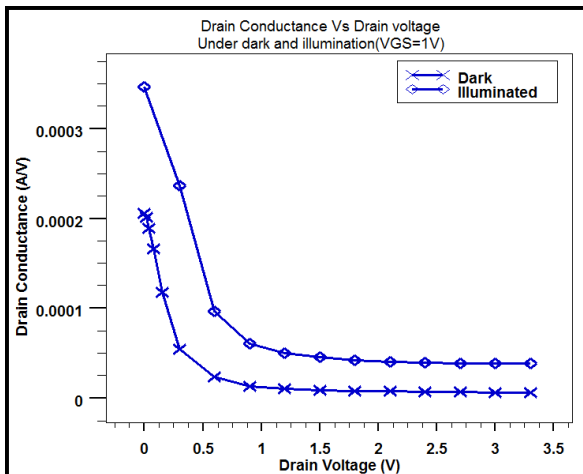

Figure $9(\mathrm{~A})$

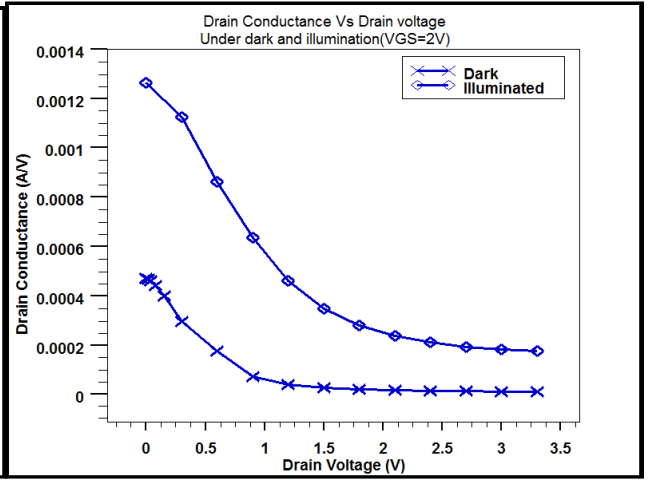

Figure 9(B)

Figure 9A. Drain Conductance Vs Drain voltage for $\mathrm{V}_{\mathrm{G}}=1 \mathrm{~V}$ in dark \& under illumination. Figure 9B. Drain Conductance Vs Drain voltage for $\mathrm{V}_{\mathrm{G}}=2 \mathrm{~V}$ in dark \& under illumination.

Figure 9A and Figure 9B are plots of drain conductance under varying voltage with $\mathrm{V}_{\mathrm{G}}$ of $1 \mathrm{~V}$ and 2 V.The graphs signify that drain conductance is almost constant with increasing drain voltage. The output conductance is proportional to the drain current, and since drain current is almost constant in saturation region of MOSFET at higher drain voltage, output conductance also becomes independent with increasing drain bias. It can be seen that drain conductance remains constant in dark as well as with photon flux radiation. Thus this verifies that optical flux modifies the effective gate bias than drain bias.

Figure 10A and Figure 10B are plots of gate transconductances at fixed drain voltage of $0.1 \mathrm{~V}$ and $1 \mathrm{~V}$ with varying gate voltage. The drain current is very small till the device enters inversion as indicated in Figure 8 Due to this the change in device gate transconductances is seen at higher gate voltage as seen in Figure 10. The transconductances curve reaches a peak and then remains almost constant. This is due to influence of $\mathrm{V}_{\mathrm{GS}}$ on effective mobility. The nature of plots of transconductance are not similar for $\mathrm{V}_{\mathrm{D}}=0.1 \mathrm{~V}$ and $1 \mathrm{~V}$. This is because the device is in linear region for $\mathrm{V}_{\mathrm{D}}=0.1 \mathrm{~V}$ and is in saturation for $\mathrm{V}_{\mathrm{D}}=1 \mathrm{~V}$. The transconductance is one of the very important factors considered in circuit design as it decides transit frequency. Thus the optically modulated device can be used to as an additional control for the device operation. 
International Journal of VLSI design \& Communication Systems (VLSICS) Vol.4, No.2, April 2013

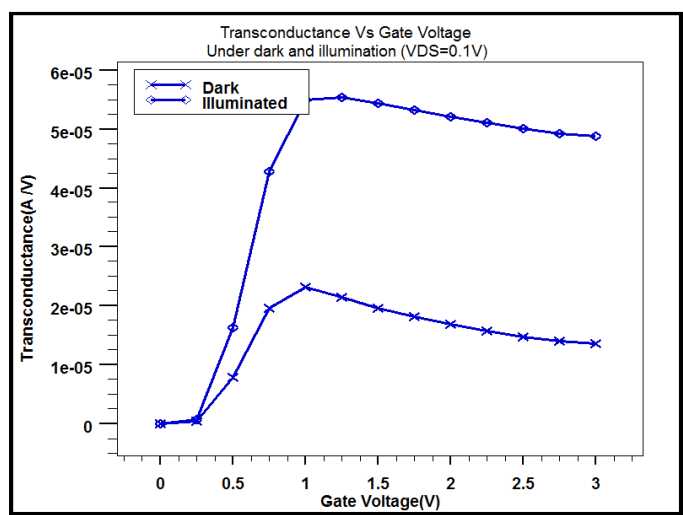

Figure 10(A)

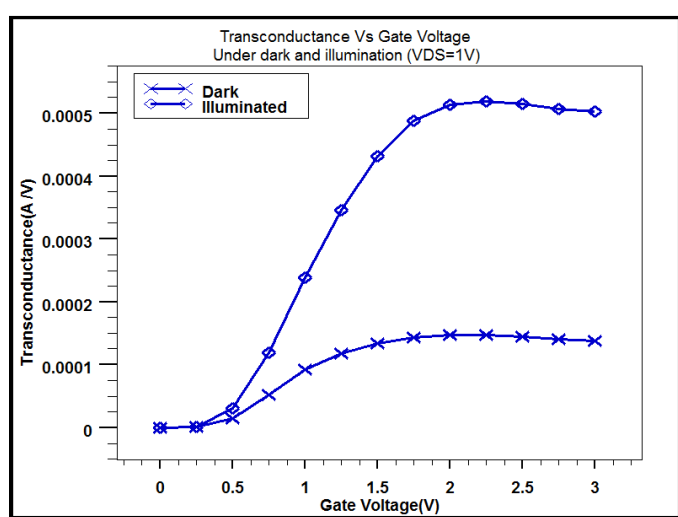

Figure 10(B)

Figure 10A: Transconductance $\mathrm{Vs}$ Gate voltage for $\mathrm{V}_{\mathrm{D}}=0.1 \mathrm{~V}$ in dark \& under illumination. Figure 10B: Transconductance $V s$ Gate voltage for $\mathrm{V}_{\mathrm{D}}=1 \mathrm{~V}$ in dark \& under illumination.
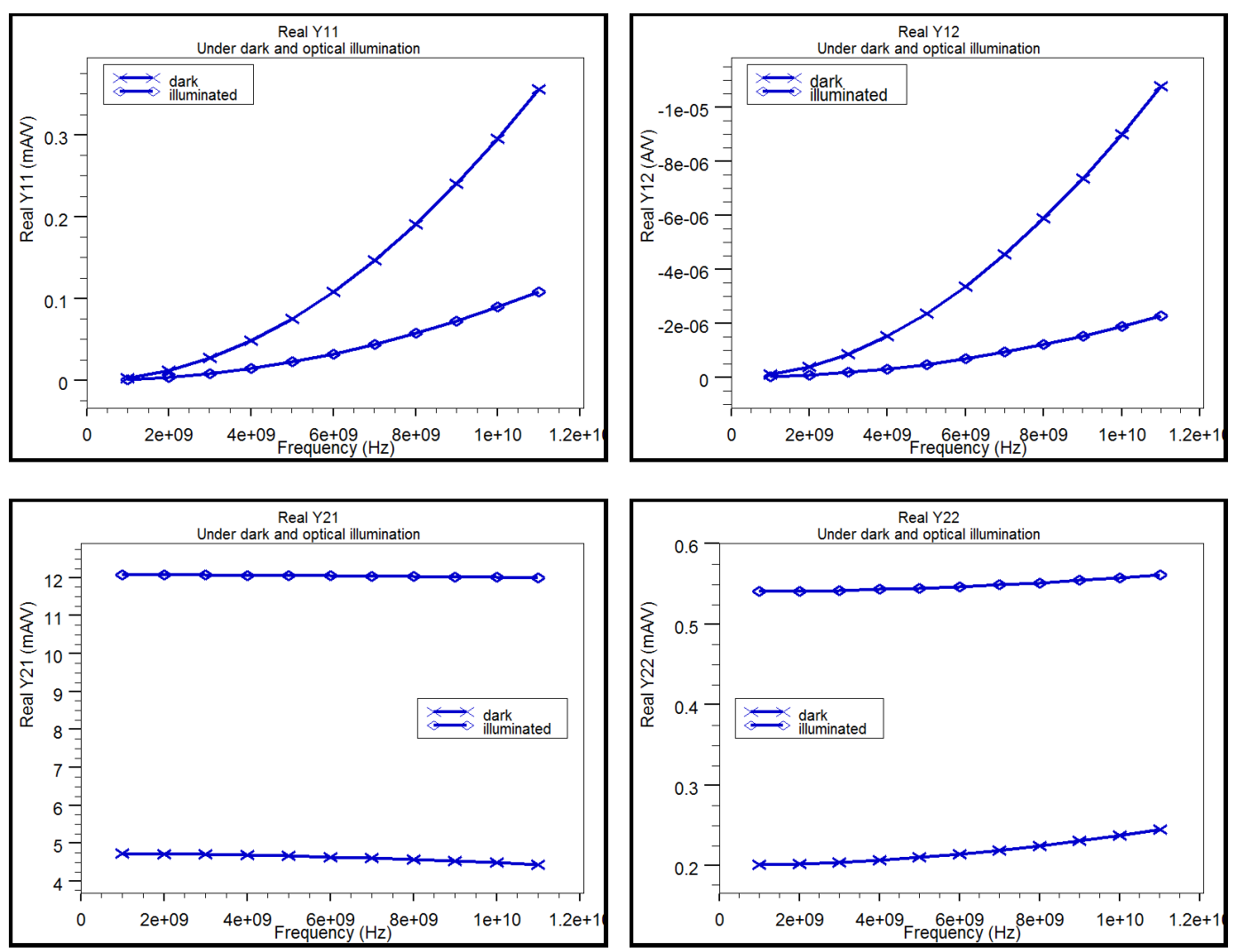

Figure 11. $\mathrm{Y}$ parameters for $\mathrm{V}_{\mathrm{G}}=1 \mathrm{~V}$ and $\mathrm{V}_{\mathrm{D}}=1 \mathrm{~V}$ in dark and under optical illumination.

Figure 11 and Figure 12 are plots of $\mathrm{Y}$ and $\mathrm{S}$ parameters for the quiescent condition of $\mathrm{V}_{\mathrm{G}}=1 \mathrm{~V}$ and $\mathrm{V}_{\mathrm{D}}=1 \mathrm{~V}$. The width of the device is necessary parameter for ac analysis. The width of the device under consideration for simulation is $50 \mu \mathrm{m}$. The frequency range for the analysis varies from $1 \mathrm{GHz}$ to $10 \mathrm{GHz}$ in steps of $1 \mathrm{GHz}$. The characteristic impedance, load impedance and source impedance for analysis are assumed to be $50 \mathrm{ohm}$ for $\mathrm{S}$ parameter and gain calculations.

Figure 11 is a plot of parameter $\mathrm{Y}_{11}, \mathrm{Y}_{12}, \mathrm{Y}_{21}$ and $\mathrm{Y}_{22}$ under dark and illuminated situation. Parameter $Y_{11}$ and $Y_{22}$ signify input and output admittance. It is seen that $Y_{11}$ and $Y_{22}$ are affected by optical radiation. For the circuit operating at RF, the input and output admittances are often 
International Journal of VLSI design \& Communication Systems (VLSICS) Vol.4, No.2, April 2013

terminated at characteristics admittance by use of matching network, hence the effect on impedance can be treated as insignificant. The parameter $Y_{21}$ signifies device forward gain or transconductance which is one of the most important parameter. The comparative plot of parameter $\mathrm{Y}_{21}$ in dark and illuminated condition signifies that illumination enhances the transconductance of the device, making it more suitable for RF operation. On other hand the plot for parameter $\mathrm{Y}_{12}$ indicates that reverse gain decreases.
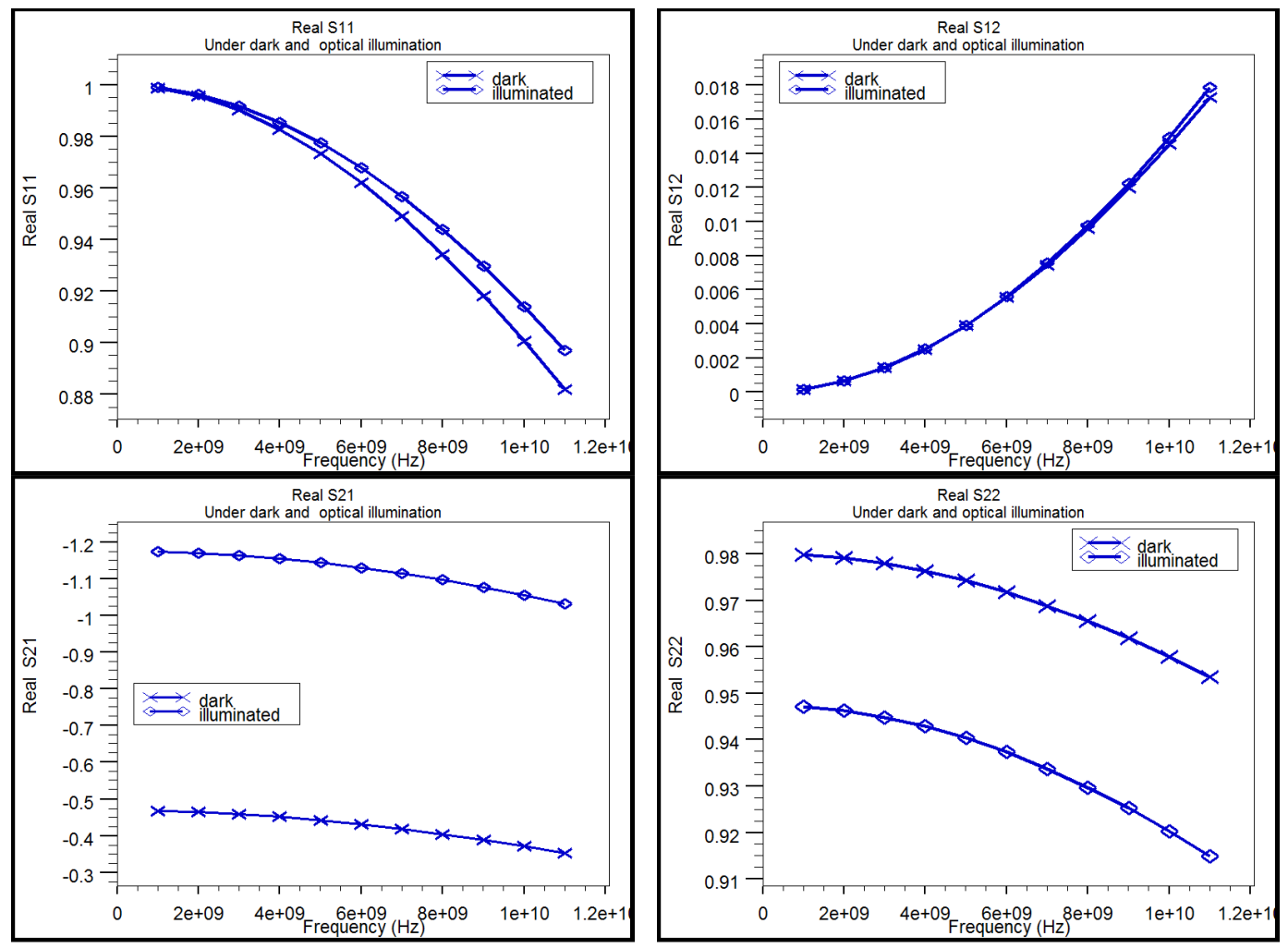

Figure 12: $\mathrm{S}$ parameters for $\mathrm{V}_{\mathrm{G}}$ and $\mathrm{V}_{\mathrm{D}}$ of $1 \mathrm{~V}$ in dark and under optical illumination

Figure 12 is a plot of parameter $S_{11}, S_{12}, S_{21}$ and $S_{22}$ under dark and illuminated situation. $S$ and $Y$ parameters for the device are inter-convertible and hence similar effect is observed for $S$ parameters. The $S_{11}$ and $S_{22}$ are related with input and output impedances and reflect similar effect as that of $Y_{11}$ and $Y_{22}$. Parameter $S_{21}$ which is associated with forward gain of the device increases with light impeachment. It can be seen that device transconductance in DC or AC always improves with optical radiation and this is reflected in parameter $S_{21}$.

Figure 13 are plots of available power gain, maximum stable power gain, maximum transducer power gain and unilateral power gain for frequency of $1 \mathrm{GHz}$ to $10 \mathrm{GHz}$ in dark and under optical illumination. The plots show that there is increase in gain under optical illumination for the frequency range under consideration. The rise of gain is consistent even with rise in frequency. The rise in gain is seen as the forward gain $S_{21}$ of the device increases, while reverse gain is almost unaffected by optical illumination, contributing to enhancement of power gain for the device under illuminated condition. 

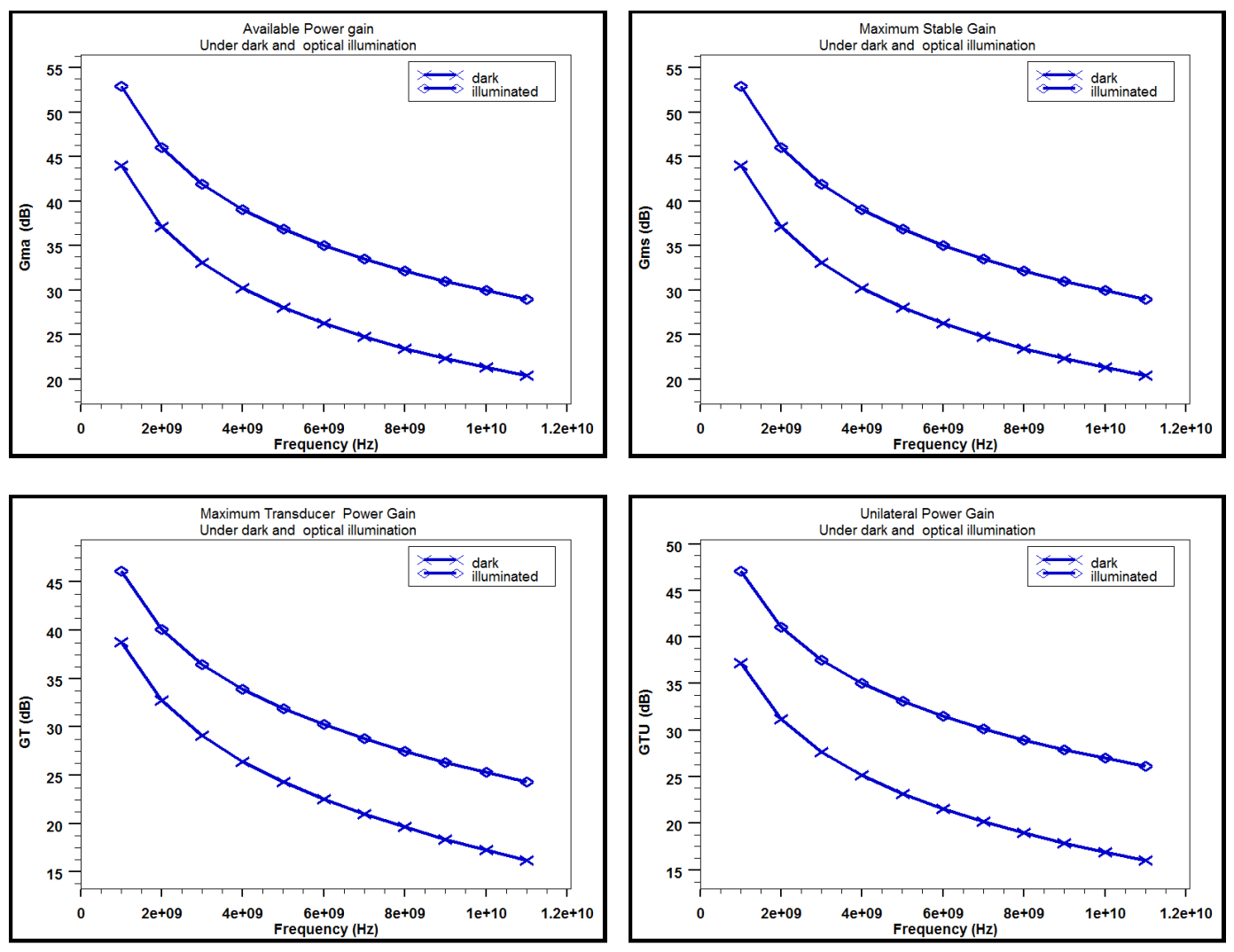

Figure 13: Power gain for $\mathrm{V}_{\mathrm{G}}$ and $\mathrm{V}_{\mathrm{D}}$ of $1 \mathrm{~V}$ in dark and under optical illumination

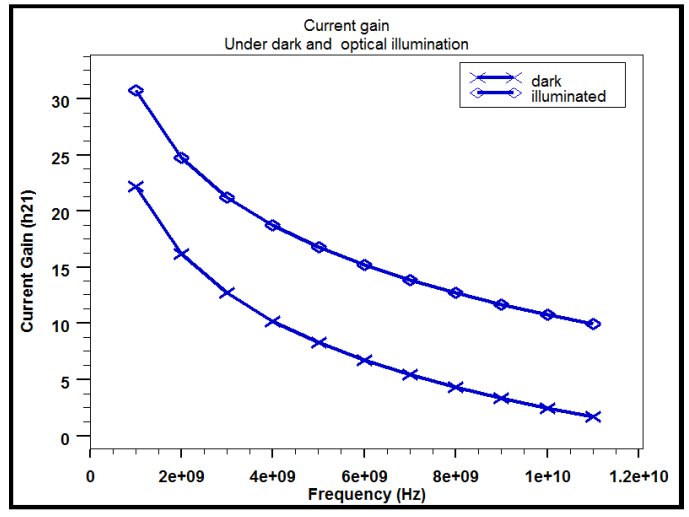

Figure 14A

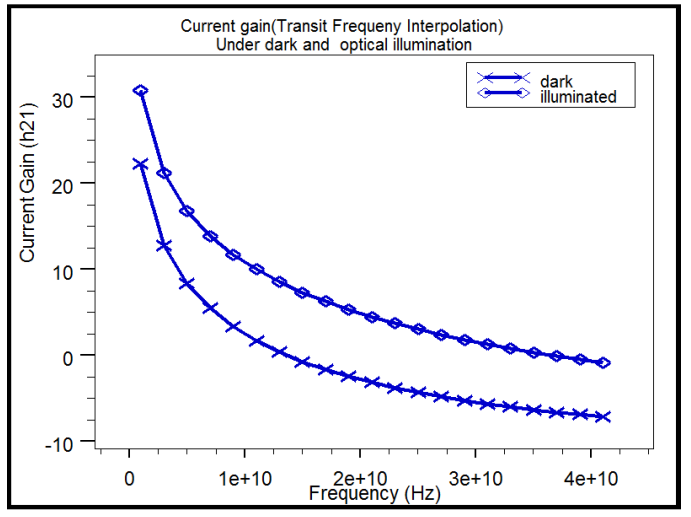

Figure 14B

Figure 14A and 14B: Current gain for $\mathrm{V}_{\mathrm{G}}$ and $\mathrm{V}_{\mathrm{D}}$ of $1 \mathrm{~V}$ in dark and under illumination

Figure 14A is plot of current gain for frequency of $1 \mathrm{GHz}$ to $10 \mathrm{GHz}$ in dark and under optical illumination. The optically illuminated MOSFET shows advancement in the current gain of the device, thus indicating improvement in the transition frequency of the device. Figure 14B is the plot of current gain for frequency of $1 \mathrm{GHz}$ to $40 \mathrm{GHz}$ in dark and under optical illumination, to locate transition frequency $\mathrm{f}_{\mathrm{t}}$. The transition frequency is the frequency at which current gain is equal to one in magnitude or $0 \mathrm{~dB}$. Using interpolation technique and extract command, the transition frequency in dark condition is found to be $13.65 \mathrm{GHz}$ and under optical radiation is $36.480 \mathrm{GHz}$. The substantial rise in transition frequency can be contributed to increase in drain current and hence improvement in transconductance. Thus significant improvement seen in 
International Journal of VLSI design \& Communication Systems (VLSICS) Vol.4, No.2, April 2013

transition frequency proves that optical radiation of the NMOSFET aids in rise of figure of merit at RF.

\section{CONCLUSIONS}

The optical response of lightly doped drain N-channel Silicon MOSFET has been studied using SILVACO TCAD software. The simulation been carried out using appropriate models and suitable numerical methods for dark condition and under optical radiation. The effect of optical illumination has been studied for $\mathrm{dc}$ and ac characteristics. The increase of drain current, conductances and transconductances of the optically illuminated device is contributed to modulation of channel conductivity due to incident photon flux.

RF performance of the device for frequency range of $1 \mathrm{GHz}$ to $10 \mathrm{GHz}$ is also investigated. The variations of admittance and scattering parameters indicate that the device capacitances are sensitive to light. This can property can be used to design optical mixers and oscillators. The improvement in transconductance and the forward current gain signify that device can be used as an optically controlled amplifier with low noise at RF. The effect on power gain and the transit frequency of the device has also been studied. The simulation results indicate prominent improvement in transit frequency and the boost in power gain of the device.

The increase in drain current, enhancement of ac and dc transconductances and the boost of power gain at RF suggest that the device is a promising candidate for optoelectronic applications at DC and RF. The present device structure is suitable for wavelength upto $1100 \mathrm{~nm}$. Modification of device structure is likely to extend the range of incident wavelength to $1500 \mathrm{~nm}$ making it suitable for long haul communication.

\section{ACKNOWLEDGEMENTS}

The authors would like to thank Department of Electronics, North Maharashtra University, Jalgaon, for providing usage of TCAD software by SILVACO international.

\section{REFERENCES}

[1] M.Jamal deen and Tor A. Fjeldly, "Preface," in CMOS RF Modelling,Characteruzation and Applications,Selected Topics in Electronic Systems, M.Jamal deen and Tor A. Fjeldly, Eds. Singapore: World Scientific Publishing Co.Pte Ltd, 2001, vol. 24, ch. Preface.

[2] Y. Cheng, "MOSFET Modelling for RF IC Design," International Journal of High Speed Electronics and Systems, vol. 11, no. 4, pp. 1007-1084, 2001.

[3] H.Zimmerman, Silicon Photodetectors and Receivers, H.Zimmerman, Ed.: Springer, 2000.

[4] Juin J. Liou and Frank Schwierz, "RF MOSFET: Recent Advances, Current status, and Future trends," Solid-State Electronics, vol. 47, pp. 1881-1895, May (2003).

[5] Jasprit Singh, Semiconductor Optoelectronics.: McGraw-Hill Inc, 1995.

[6] Dana Cristea, "Silicon Opto-FET for Photonic Integrated Circuits," in EDMO 2001, Vienna, 2001, pp. 273-279.

[7] J.D. SCHAUB et al., "Lateral PIN photodiodes for high-speed silicon optical receivers," IEEE, Industrial Research Highlights 2002.

[8] Chu-Hsuan Lin and Chee Wee Liu, "Review Metal-Insulator-Semiconductor Photodetectors," Sensors, vol. 10, pp. 8797-8826; September 2010.

[9] Abid Kamran, "Gated Lateral Silicn P-I-N junction photodiodes," University of Glasgow, PhD thesis 2011.

[10] Jaejune Janguse, "Small Signal Modelling of RF CMOS," Stanford University, PhD Thesis 2004.

[11] Prerana Jain, Mishra B.K., and Gayatri Phade, "Power Gain Analysis of Optically Illuminated MOSFET," International Journal Of Computer Applications, vol. 51, no. 16, pp. 50-54, August 2012. 
[12] G.A.Amstrong and C.K.Maiti, TCAD Design for $\mathrm{Si}, \mathrm{SiGe}$ and GaAs Integrated Circuits. London, U.K.: IET, 2007.

[13] Jain Prerana and Mishra B.K., "An Invesigation of DC characteristics in Multifinger Optically Illuminated MOSFET," International Jornal of Computer Applications, vol. 61, no. 2, pp. 12-17, January 2013.

[14] Jain Prerana and Mishra B.K., "CV Investigation in Optically Illuminated MOSFET," International Journal of Engineering Research and Applications, vol. 47, pp. 1881-1885, Nov-Dec 2012.

[15] Prerana Jain, Mishra B.K., and Phade Gayatri, "AC performance of Optically Controlled MOSFET," in SCEECS, Bhopal, 2012.

[16] "Athena USER manual," 2010.

[17] "ATLAS User Manual," 2010.

[18] Christopher M. Snowden, Semiconductor Device Modelling, 1st ed. Oxford, Great Britain: Springer Veralag, 1989.

[19] Bert Kerr, Carl L. Axness, Jason C. Verley, Charles E. Hembree, and Eric R. Keiter, "A new timedependent analytic model for radiation induced photocurrent in finite 1D epitaxial diodes," Sandia National Labrotaries, 2012.

[20] X. Li, W. Wu, H. Wang, A. Jha, R. van Langevelde, G.D.J. Smit, A.J. Scholten G. Gildenblat, "PSP: An Advanced Surface-Potential-Based MOSFET Model for Circuit Simulation," IEEE Trans. Electron Dev, vol. 53, no. 9, pp. 1979-1993, 2006.

[21] D. Vasileska, D. Mamaluy, H. R. Khan, K. Raleva, and S. M. Goodnick, "Semiconductor Device Modeling," Journal of Computational and Theoretical Nanoscience, vol. 5, pp. 1-32, 2008.

[22] Jörgen Olsson, "SILICON RF-DEVICES," Top Amplifier Research Groups in a European Team, Sweden, Tutorial 2004.

[23] Reinhold Ludwig, RF Circuit Design, Theory and Applications. New Jersey: Prentice Hall, 2000.

[24] Prerana Jain, B.K. Mishra, and Phade G., "S parameters of Optically Illuminated MOSFET," in International Conference on Advancements in Engineering and Management(ICAEM), Hyderabad, 2012.

\section{Authors}

Dr.B.K.Mishra was born in $5^{\text {th }}$ June 1966,in Bihar He completed his B.E. in Electronics Engg in 1988 and M.E. in Electronics and Communication Engg in 1992. He was awarded PhD degree from Birla Institute of Technology in 1998. He has 23years of teaching experience. His present research interest focuses on device and structures working at microwave frequencies and optical sensors. He is presently working as Principal at Thakur College of Engineering and Technology, Kandiwali, Mumbai..

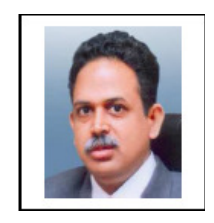

Prerana Jain born on $30^{\text {th }}$ Nov 1969, completed her graduation in Electronics and Telecommunication in 1991 from Pune University. She completed her Mtech in VLSI Technology from North Maharashtra University in 2007 and has work experience of 20 years in industry and teaching. Presently she is pursuing $\mathrm{PhD}$ under guidance of Dr.B.K.Mishra. Her research area is semiconductor device modelliing for optoelectronic applications. She is presently working as Asst Prof in North Maharashtra university,

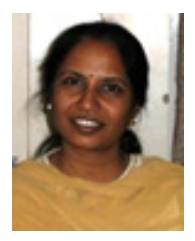
Jalgaon. 